\title{
Electrical Impedance Methods in Neuromuscular Assessment: An Overview
}

\author{
Seward B. Rutkove and Benjamin Sanchez \\ Department of Neurology, Beth Israel Deaconess Medical Center, Harvard Medical School, Boston, \\ Massachusetts 02215 \\ Correspondence: srutkove@bidmc.harvard.edu
}

Electrical impedance methods have been used as evaluation tools in biological and medical science for well over 100 years. However, only recently have these techniques been applied specifically to the evaluation of conditions affecting nerve and muscle. This specific application, termed electrical impedance myography (EIM), is finding wide application as it can provide a quantitative index of muscle condition that can assist with diagnosis, track disease progression, and assess the beneficial impact of therapy. Using noninvasive surface methods, EIM has been studied in a number of conditions ranging from amyotrophic lateral sclerosis to muscular dystrophy to disuse atrophy. Data support that the technique is sensitive to disease status and can offer the possibility of performing clinical trials with fewer subjects than would otherwise be possible. Recent advances in the field include improved approaches for using EIM as a "virtual biopsy" and the development of combined needle impedance-electromyography technology.

\section{APPROACHES FOR NEUROMUSCULAR ASSESSMENT: THE UNDERLYING MOTIVATION AND AN OVERVIEW OF CURRENT TECHNOLOGIES}

The neuromuscular disorders include condi-

tions that affect the peripheral nerve, extending up to the anterior horn of the spinal cord and the dorsal root ganglia, the neuromuscular junction, and the muscle itself (Preston and Shaprio 2005). They include problems ranging from the very mild, such as disuse atrophy associated with orthopedic injury or exposure to microgravity, to very severe, debilitating conditions such as Duchenne muscular dystrophy (DMD) and amyotrophic lateral sclerosis (ALS). For clinicians and researchers alike, a major challenge remains the ability to promptly diagnose these conditions and to assess deterioration and response to therapy.

For the most part, standard clinical assessment tools such as the physical examination, strength, and functional testing, and basic laboratory tests can provide substantial insights into the nature of a given condition, quickly helping the physician appropriately categorize and potentially diagnose a condition. The recent advent of relatively low-cost next-generation genetic sequencing has now also greatly improved our ability to accurately diagnose a variety of hereditary diseases. In some cases, a muscle or nerve biopsy is still needed to help definitively identify a condition. Together, these tools can also be used to evaluate disease progression and the effect of therapy; however, they are often sub-

Editors: Valentin A. Pavlov and Kevin J. Tracey

Additional Perspectives on Bioelectronic Medicine available at www.perspectivesinmedicine.org

Copyright (C) 2019 Cold Spring Harbor Laboratory Press; all rights reserved; doi: 10.1101/cshperspect.a034405

Cite this article as Cold Spring Harb Perspect Med 2019;9:a034405 
stantially limited in achieving the necessary sensitivity for use in clinical drug trials. In addition, invasive techniques that would need to be repeated possibly several times over a period of several months during clinical trials or during standard patient care are undesirable. Moreover, these tools provide limited insight into the mechanical aspects of contraction, muscle's major biological function.

Thus, a variety of supplementary tools exist. Two of the oldest and most entrenched technologies are standard nerve conduction studies (NCSs) and needle electromyography (EMG) (Kimura 2001; Preston and Shaprio 2005). In NCSs, a specific nerve is electrically stimulated and the response from the nerve itself or from an associated muscle is measured; both the amplitude of the response and the calculated conduction velocity provide information into nerve function and health. In EMG, a small needle containing one (i.e., monopolar) or two (i.e., concentric) electrode(s) is placed in a muscle and gently maneuvered to assess myofiber irritability, namely, how easily electrical discharges are produced. The patient is then asked to contract the muscle and the morphology and firing pattern of motor unit action potentials recorded. Alterations in the size and shape of the potentials and their firing pattern indicate the presence of disease pathology, with primary neurogenic abnormalities and primary myopathic abnormalities generally being readily distinguishable from one another.

In addition to EMG, both magnetic resonance imaging (MRI) and ultrasound technologies are also being increasingly applied to the assessment of neuromuscular disease in the past few years (Simon et al. 2016). Although both can be used to assess localized nerve lesions including tumors, in most cases, these technologies are being used to assess muscle condition and change over time and are not serving as a primary diagnostic. With MRI, this includes evaluating cross-sectional area and percent fat in a given region of the muscle (Willcocks et al. 2016); MR spectroscopy can also be used to evaluate metabolic changes in muscle both at rest and during exercise (Kemp et al. 2015). With ultrasound, muscle echointensity is measured as a marker of disease damage, with it increasing in most diseases (Zaidman et al. 2017); muscle thickness can also be assessed (Caresio et al. 2017).

However, all of these "standard" techniques have limitations. For example, EMG relies on subjective interpretation. Although there are quantitative methods available, they are not widely used because of their time-consuming nature. Although nerve conduction methods are easier to quantify, obtaining high repeatability, especially with sensory studies, can be challenging because the waveform amplitude can change dramatically depending on electrode location. Similarly, MRI has a number of limitations hindering its application in the clinic, including its requiring patients to go to a specialized facility, its being challenging to use in young children without sedation, its requiring patients to lie flat (a problem in patients with neuromuscular respiratory compromise), and its being costly and rather time consuming to perform. Ultrasound is easier to perform than MRI; however, its limitations include that small alterations in probe angle greatly distort image quality, proprietary algorithms for echo propagation and measurement create system-tosystem variability, and attenuation of the signal in diseased muscle prohibits its assessment of deeper muscles.

The application of electrical impedance to the assessment was motivated by the limitations presented by all of these technologies. As will become clear below, the strengths of using impedance techniques for the assessment of neuromuscular disease includes its ease of use, its intrinsic quantitative nature, flexibility of application, high repeatability, sensitivity to muscle deterioration, and response to therapy.

\section{ELECTRICAL IMPEDANCE METHODS IN MEDICINE: AN OVERVIEW}

The study of biological tissues' electrical properties started in the 18th century with the experiments performed by Luigi Galvani. However, it was not until 100 years later that James C. Maxwell established the laws of electromagnetism and that researchers had the tools to un- 
Electrical Impedance in Neuromuscular Assessment

derstand how materials interacted with electric and magnetic fields. The theory of electromagnetic fields led in the subsequent decades to the broader development and application of electrical impedance methods in physical and biological sciences.

Since the mid- to late 20 th century, electrical impedance methods have found value in a variety of medical applications. Electrical impedance has been extensively used to estimate volumes of different tissues or fluids within the body (Ellis 2000; Kyle et al. 2004). Electrical impedance can also be used to study blood circulation, an application also known as impedance cardiography (ICG) (Kubicek et al. 1970). Today, ICG has been adopted as a noninvasive way to monitor cardiac output in the physician's office and hospital setting. Other well-established uses include neonatal lung monitoring (Brown 2003), tumor imaging (Toso et al. 2000; Phillips 2013), and assessment of skin lesions (Aberg et al. 2003).

Closely connected to these practical medical applications, electrical impedance methods can also be applied for more basic scientific purposes, such as determining the actual electrical properties of tissues and fluids (Fricke and Morse 1925; Cole 1928; Schwan and Kay 1957; Geddes and Baker 1967; Stoy et al. 1982; Foster and Schwan 1989). As discussed further below, the pathophysiologic process of any disorder will alter its passive electrical properties and can serve as a useful biomarker of disease status thus providing a direct measure of tissue condition. For example, edema in the muscle as observed in muscle injury with increased T2 signal on MRIs will increase the effective conductivity of the muscle. In contrast, the deposition of fat will decrease tissue-apparent conductivity.

In short, although electrical impedance concepts have been understood for nearly 100 years (Schwan 1999), the medical applications of the technologies have been only realized over the last few decades, in part because of both the technical and theoretical challenges involved. It is likely for this reason, and also because the standard electrophysiological approaches of NCSs and EMG have been fairly successful, that this field had not matured earlier.

\section{UNDERLYING CONCEPTS OF ELECTRICAL IMPEDANCE MEASUREMENTS, MUSCLE'S ANISOTROPIC ELECTRICAL PROPERTIES}

Most bioelectrical impedance methods rely on a four-electrode impedance measurement to avoid electrode artifacts that would occur if only two electrodes were used (Schwan 1968). One method to measure impedance consists of applying an alternating electrical current via a pair of electrodes (the high- and low-current electrodes) and then measuring the resulting voltage via a second pair of electrodes (the high- and low-voltage electrodes). There are also more accurate measurement approaches, for example, in which both the current and the voltage signals are measured (Sanchez et al. 2013a,b, 2015b). Tissue's electrical properties impact the flow of electrical current thus determining the features of the voltage generated, namely, the amplitude and the time lag with respect the applied current. The change in amplitude is associated with the resistive behavior of the tissue, that is, the passive compositional structures including free water, connective tissue, and fat; whereas the time lag is related to the capacitive/reactive components, mainly the myofiber membranes, which act as distributed capacitors. Both the resistive and reactive elements determine the complex impedance, a term coined by Oliver Heaviside, whose standard engineering notation is $Z=R+j X$ (in Ohm units), where $R$ and $X$ are the resistance and reactance components and $j$ represents the complex imaginary unit to distinguish both components. Alternatively, one can define the impedance through its magnitude $|Z|=\operatorname{sqrt}\left(R^{2}+\right.$ $\left.X^{2}\right)$ and phase angle $\theta=\arctan (X / R)$, the latter associated to the cellular integrity. For additional background on these basic impedance concepts, we refer the reader to any standard introductory physics textbook. Importantly, only two parameters are necessary to define the impedance, typically the resistance $(R)$ and the reactance $(X)$ are measured simultaneously, from where the impedance magnitude $(Z)$ and phase $(\theta)$ can be easily calculated and vice versa.

By nature, impedance values contain the contribution of additional factors beyond the 
inherent electrical properties of muscle, namely, the conductivity and relative permittivity. These additional factors include the electrodes' characteristics (e.g., electrodes' size and material), electrodes' arrangement (e.g., electrodes' distance and shape), and conductor volume (i.e., volume of the limb and position). However, when collected in a standardized fashion, impedance data can provide useful outcomes, for example, to assess disease progression. Still, it is also possible to disentangle the electrical properties of a biological tissue or fluid from the impedance values. This is a more challenging task to perform because it requires interdisciplinary skills using both theoretical and experimentally based knowledge from several disciplines, including mathematics, physics, and engineering. Another complexity when measuring the electrical properties of muscle is to assess their directional dependence, a concept known as anisotropy. This phenomenon is very prominent in muscle given its highly organized cellular and fascicular structure and thus current flows more easily along the fibers than across them (Epstein and Foster 1983). Assuming the electrodes are actually in direct contact with the muscle, then the anisotropy ratio can be approximated as follows (Rush 1962):

$$
0<\left(\frac{R_{L}}{R_{T}}\right)^{2} \leq 1
$$

where $R_{L, T}$ are the resistance of the impedance measured with the electrode probe in contact with the muscle oriented in the longitudinal (L) (along the muscle fibers) and transverse $(T)$ (across the muscle fibers) directions (Sanchez et al. 2014a). A muscle with a greater difference between the longitudinal and transverse resistance will have a smaller anisotropy ratio, with 1.0 being the maximum of the anisotropy ratio for a completely isotropic material in which the propagation of the current does not depend on its direction. Simply altering the voltage and current electrodes' orientation with respect to the major muscle fiber direction is one approach for assessing in situ muscle electrical anisotropy but there are more sophisticated approaches (Kwon et al. 2017a). As noted below, this is likely one feature of muscle that is altered in disease states.

In addition to anisotropy, another unique feature of skeletal muscle tissue is its ability to contract and generate force. Studies have shown that impedance can be used to detect changes between relaxed and contracted muscle (Bozler 1935; Bozler and Cole 1935; Zagar and Krizaj 2008), thus offering the prospect of its being able to reveal disease dependencies (Shiffman et al. 2003; Sanchez et al. 2014b). However, unless only isometric contractions are performed, these measurements can be experimentally difficult as geometric changes in the muscle or limb that occur when going from the state of relaxation to contraction will impact the data recorded and hinder its interpretation. Approaches for dealing with this challenge, including the use of impedance imaging techniques as described in the last section, are in development.

\section{OVERVIEW OF THE CLINICAL APPLICATION OF ELECTRICAL IMPEDANCE MYOGRAPHY IN NEUROMUSCULAR DISORDERS}

\section{EIM Methods and Applications}

Various electrical impedance myography (EIM) methods have been applied in the nearly 20 years since the technique's introduction (Fig. 1). Early approaches used off-the-shelf impedance systems or bioimpedance systems intended for body composition measurement, with adhesive electrodes that were manually placed on the hands (for electrical current application) and on the skin overlying a muscle group of interest (for voltage measurement) (Sanchez et al. 2016). The technology was improved in that custom commercial impedance devices became available with different size, disposable electrode arrays to test a variety of muscles (Zaidman et al. 2015; Kolb et al. 2016; Shefner et al. 2018).

EIM has also been applied in various animal studies as models of human disease; mice (Li et al. 2012), rats (Ahad and Rutkove 2009), and dogs (Hakim et al. 2017) have been measured using adhesive electrodes, surface metal electrodes, and needle electrodes. The specifics of which electrode is used depends on the size of 

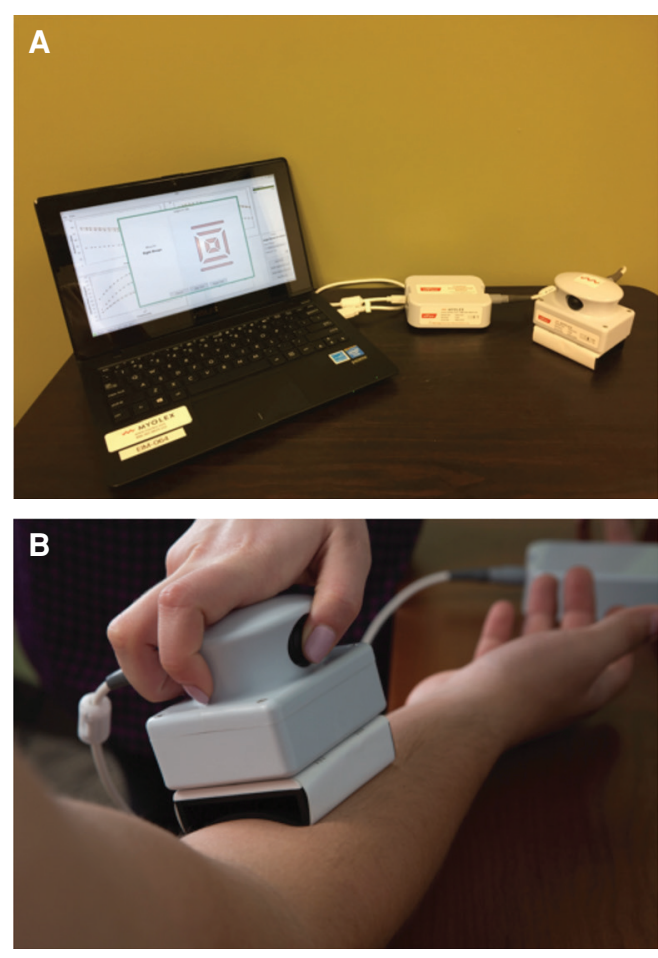

Figure 1. Electrical impedance myography (EIM) system (Myolex). (A) EIM consists of three components: a standard laptop, a power convertor, and the impedance measuring device itself with an attached electrode array. (B) EIM being performed on the forearm flexors.

the animal, the presence of fur, and the specific questions of interest. The potential application of EIM as a primary veterinary tool to assess animal muscle condition also remains on the horizon. To some extent, this is coming full circle, because a long-standing use of basic impedance techniques has been in the assessment of the relative leanness of animal meat intended for human consumption (Marchello and Slanger 1992).

One additional point of importance to underscore is that a variety of impedance variables are simultaneously acquired with any EIM measurement (reactance, resistance, phase, and the impedance itself). Moreover, the directional dependence ( parallel to the long axis of the muscle versus perpendicular to it) and the specific frequencies used add further complexity. Thus, much effort has been expended on evaluating
Electrical Impedance in Neuromuscular Assessment

which impedance outcomes are most relevant to a specific disease. In the following clinical review, we provide the EIM data that appear to be most promising for a given disorder, but it is important to underscore that this remains an active area of research.

\section{EIM as a Primary Diagnostic Technique}

A variety of studies have shown that EIM using surface electrodes can differentiate healthy from diseased muscle. This has been shown in ALS (Tarulli et al. 2009b), spinal muscular atrophy (SMA) (Rutkove et al. 2010), radiculopathy (Spieker et al. 2013), muscular dystrophies, including DMD (Rutkove et al. 2014), and facioscapulohumeral muscular dystrophy (FSHD) (Statland et al. 2016). However, in most of these cases, simple group differences were shown, and EIM's diagnostic accuracy was not specifically assessed. In fact, as might be anticipated, most often there is substantial overlap between healthy and diseased values, especially early in the disease course, although differences become more obvious as the disorder advances.

As for differentiating across disease types (in comparison to differentiating diseased from healthy individuals), a simple question is whether EIM can differentiate between primary nerve disease impacting muscle (neurogenic disease), primary muscle disease (myopathic disease), and disuse change. A study of ex vivo mouse muscle from healthy, ALS, DMD (via the $m d x$ mouse), and disuse atrophy mice (produced via hindlimb suspension), showed that the conductivity alone could differentiate to between these conditions (Li et al. 2014). In addition to this mouse work, one human study found that the directional dependence in surface EIM values could also distinguish healthy versus diseased muscle (Garmirian et al. 2009). More recently, a study sought to evaluate EIM's potential use as a means of discriminating ALS patients from those with mimicking disorders and from healthy individuals (Shefner et al. 2018). The test was effective at distinguishing healthy from ALS showing good accuracy with a receiver operating characteristic (ROC) area under the curve (AUC) of 0.80 , but was of limited use 
in distinguishing ALS patients from disease mimics (ROC AUC $=0.62$ ), which included conditions such as polyradiculopathy, polyneuropathy, and myasthenia gravis.

EIM as a Surrogate Measure of Disease Severity, Progression, and Response to Therapy

EIM's greatest long-term value will be in the assessment of individuals over time, as a method of assessing disease progression and response to therapy. Hence, we provide additional detailed overview of this potential application of EIM technology in a variety of different medical contexts.

\section{Neurogenic Disorders}

Amyotrophic Lateral Sclerosis. EIM's capability to sensitively track ALS progression has been studied at least three times (Rutkove et al. 2007, 2012a; Shefner et al. 2018), with the last being a multicenter study, using a dedicated EIM system that showed a greater than fivefold improvement in sensitivity to progression than standard clinical measures (Shefner et al. 2018). EIM has also been used to assess bulbar dysfunction by measuring the tongue (McIlduff et al. 2015; Shellikeri et al. 2015); this application may be especially relevant because there are few reliable bedside tools that can quantitatively assess this body region.

Parallel investigative efforts using ALS mouse and rat models helped to establish the relationship between EIM and standard, accepted neurophysiological measures, including the compound motor unit action potential amplitude and the motor unit number estimate (Wang et al. 2011; Li et al. 2014).

Spinal Muscular Atrophy. Longitudinal studies have shown that in healthy children, the 50$\mathrm{kHz}$ impedance phase gradually increases over time, likely because of myofiber growth and maturation (Rutkove et al. 2012b). EIM in SMA, in contrast, shows relative stability of the phase value over time consistent with the absence of normal myofiber growth that in SMA is associated with a reduced motor neuron complement in older children (Rutkove et al. 2012b).
EIM has also been shown to correlate to disease severity (Rutkove et al. 2010). Similarly, a multicenter study in infants with type 1 SMA showed EIM's ability to distinguish healthy children from SMA at baseline (Kolb et al. 2016) and over time (unpubl.). EIM's sensitivity to treatment effect in SMA has also been shown using antisense oligonucleotides in the SMA $\Delta 7$ mouse model (Arnold et al. 2016).

Nerve Injury and Radiculopathy. EIM can be used to help facilitate the diagnosis of radiculopathy by performing side-to-side comparisons (Rutkove et al. 2005; Spieker et al. 2013), achieving an accuracy similar to that of needle EMG (Spieker et al. 2013). In rat models of sciatic crush, EIM values mirrored standard neurophysiologic approaches (Ahad et al. 2010), with decreased impedance phase after injury that gradually returned to baseline over time. One investigation also showed EIM's capability of discriminating among different traumatic nerve injuries in the upper extremity ( $\mathrm{Li}$ et al. 2017b).

Carpal Tunnel Syndrome. EIM has also been used to evaluate carpal tunnel syndrome (CTS), the most common compression neuropathy. One study showed that EIM values of the affected abductor pollicis brevis muscle in CTS patients are lower than those for healthy subjects, with no significant differences in unaffected muscles (Li et al. 2017a).

Other Neurogenic Disorders. EIM is currently being used as a potential biomarker of disease progression and drug efficacy in studies of polyneuropathy, including Charcot-MarieTooth disease and giant axonal neuropathy; however, to our knowledge there is no published literature assessing these applications. Also, as far as we know, it has not been used in diseases of the neuromuscular junction, such as myasthenia gravis and Lambert-Eaton myasthenic syndrome.

\section{Myopathic Conditions}

Duchenne Muscular Dystrophy. An initial study showed that $50 \mathrm{kHz}$ phase is lower in DMD than in healthy boys (Rutkove et al. 2014). Further, clinical studies have found EIM phase is sensitive to disease progression in boys of all ages; moreover, it is also sensitive to treat- 
ment effect using steroids (Rutkove et al. 2017b). A second confirmatory study supports this basic concept (Zaidman et al. 2018). In the $m d x$ muscular dystrophy mouse model, EIM resistance and reactance values were lower, with correlation of EIM values to actual connective tissue content (Li et al. 2013a). This relationship was also supported by $\mathrm{T} 2$ signal intensity on MRI in a separate study (Wu et al. 2015). EIM has also shown sensitivity to the impact of myostatin inhibition in both wild-type and $m d x$ mice (Sanchez et al. 2015a); an additional recent study also showed its potential for detecting dose-dependent effects of myostatin inhibition (Nagy et al. 2018).

Facioscapulohumeral Muscular Dystrophy. EIM reactance values in patients with FSHD were found to correlate with the degree of abnormality on MRIs (Statland et al. 2016). More recently, a study has shown that sample size requirements for a potential clinical therapeutic trial in FSHD could be substantially reduced using EIM (Mul et al. 2018). Because EIM can be flexibly used at the bedside to evaluate affected muscles, it could be especially useful in this disease, which is characterized by remarkable variability in presentation.

Myotonic Dystrophy. Limited studies in both myotonic dystrophy type 1 and 2 patients have been performed by our group (unpubl.). Similarly, some data has also been collected in mouse models of DM1 (unpubl.), supporting EIM's capability to detect both the presence of the disease and potentially the effect of therapy.

Inflammatory Myopathies. Impedance phase has been observed to be reduced in individuals with inclusion body myositis, likely because of the presence of more resistive fat tissue in the quadriceps muscle (Rutkove et al. 2002). In addition, impedance phase correlated to the clinical status of patients with polymyositis and dermatomyositis (Tarulli et al. 2005).

\section{Sarcopenia, Disuse, and Cachexia}

EIM could be of especial value in these groups of disorders, because standard electrophysiological studies such as EMG is insensitive to the associated pathology of these disorders (Urso et al. 2006).
Sarcopenia. EIM phase values decrease with increasing age, as shown in a cross-sectional study of 100 subjects across the age spectrum (Aaron et al. 2006). Specifically, a nonlinear relationship was found between EIM $50 \mathrm{kHz}$ phase in the tibialis anterior and gastrocnemius, with marked reduction with age in both sexes for people over 60 years. Longitudinal measurements on four patients over 75 years of age without any superimposed neuromuscular condition also showed a decline over time. Reduction in the $50 \mathrm{kHz}$ reactance was found to be more evident in a subsequent cross-sectional study with greater change in men than women (Kortman et al. 2013). Both studies were in good agreement with previous work showing that other markers of sarcopenia were more prominent in men than women (Iannuzzi-Sucich et al. 2002), and that changes in lower extremities were more substantial than in the upper extremities (Janssen et al. 2000). In addition, impedance in the lower extremities of older adults has been found to correlate with function measures such as force (unpubl.).

Disuse Atrophy. In ankle fracture patients, impedance phase gradually increased after cast removal and return to full weight bearing, consistent with recovery (Tarulli et al. 2009a). Analogous observations were found in the hind limbs of rats undergoing hind-limb suspension, a standard model for disuse (Li et al. 2013b). After 2 weeks of suspension, impedance phase dropped by $33 \%$. Two weeks after returning to normal weight bearing, the animals had partially recovered being 19.5\% lower than baseline. The effect of microgravity on muscle was also studied aboard the NASA STS-135 Space Shuttle mission in 2011; this study showed significant reductions in impedance phase. In this study, however, impedance phase were on average $43 \%$ lower than the control group (Sung et al. 2013).

\section{Traumatic Injury of Muscle}

Muscle traumatic injury has been also studied in elite soccer players and confirmed with ultrasound and MRI (Nescolarde et al. 2013, 2015). Muscle injuries included hematoma caused by 
trauma and muscle tears of different severities in the lower extremity muscles. The EIM values on the injured side decreased with injury and gradually recovered over time. These findings support the concept of using impedance approaches as an easily applied biomarker to help assist in determining readiness to return to play (Nescolarde 2017).

Finally, muscle injury induced by eccentric contractions has also been studied in $m d x$ and healthy mouse quadriceps muscle (Sanchez et al. 2017). Reductions in impedance phase were observed over a period of 48 hours, likely related to the presence of edema as confirmed by increased T2-signal on MRI.

\section{ONGOING AND NEW DIRECTIONS}

\section{Muscle Impedance Imaging}

By using multiple electrodes and switching the pair of current and voltage electrodes, it is possible to obtain an image of the conductivity distribution of an object. This field is known as electrical impedance tomography (EIT) and was started 40 years ago (Holder 2004). EIT has had its most substantial clinical success in imaging and quantifying lung volumes (Adler et al. 2012) and also for brain monitoring (Holder 1992). Nevertheless, impedance imaging can also be applied to muscle (Silva et al. 2014), helping to eliminate the contribution of subcutaneous fat on the measured impedance values greater than can be accomplished with a surface, four-electrode impedance measurement (Rutkove et al. 2017c; Kwon et al. 2018b). This technique can also potentially assist in assessing muscle contraction (Rutkove et al. 2017a), because the geometric alterations associated with contraction can be taken into account.

\section{Extending EIM as a Virtual Muscle Biopsy}

Applying impedance methodologies along with predictive modeling can make it possible to identify muscle biopsy features without the need for actual excised muscle tissue. One application is the prediction of myofiber size, because the reactance frequency dependence is sensitive to the radii of myofibers. In one recent study, EIM was applied to young mice of varying age from postnatal day 5 through day 35, using a small four-electrode needle array (Kapur et al. 2018 b). Because change in myofiber size was the only major histological alteration over this time, with myofiber area increasing approximately eightfold, a combination of EIM parameters could predict myofiber size with errors of no more than $18.40 \%$. Moreover, a study in ALS mice of varying age and varying degrees was able to accurately predict the correct mean myofiber size as well as the variability of fiber size in a given animal (Kapur et al. 2018a). We are currently investigating whether this approach can also be used for estimating extracellular abnormalities (e.g., fat and connective tissue deposition) as well as intracellular abnormalities (e.g., abnormal glycogen accumulation and mitochondrial proliferation).

\section{Needle Impedance Electromyography}

As noted above, needle EMG is one of the standard diagnostic tests for the assessment of neuromuscular conditions. However, EMG is limited to evaluating the "active" electrical properties of myofibers, namely, the depolarization of electrically active myofiber membranes, either spontaneously or the result of voluntary contraction. In contrast, impedance is mainly sensitive to the nonelectrically active properties of the material including its composition and structure. To date, most EIM approaches have focused on using surface electrodes for ease of use in patients, and it is not obvious how to combine both needle EMG and surface EIM modalities simultaneously. Nevertheless, by merging the two technologies, it could help to provide additional insight into abnormal myofiber electrical properties in disease.

Thus, we have recently moved the development of EIM technology into a new direction by using impedance as a valuable add-on to standard needle EMG, creating an impedance-EMG needle (Fig. 2). Simulation and pilot experimental studies support the premise of this technique (Kwon et al. 2017b). Because impedance can be recorded simultaneously with standard needle 


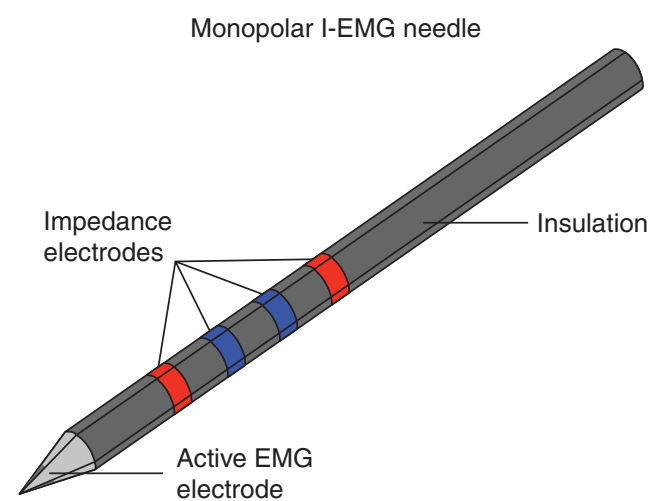

Figure 2. Sketch of proposed impedance-electromyography (I-EMG) needle. The red electrodes are the impedance current emitting electrodes and the blue are for voltage measurement. The dark gray tip represents the active EMG electrode that would be referenced against a surface electrode with a ground electrode.

EMG (Kwon et al. 2018a), needle impedanceEMG could expand the diagnostic value of needle EMG with minimal added effort and expense. Taking this concept one step further, to help the clinician to identify other abnormalities within the muscle, we have also evaluated the feasibility of imaging with impedance using a needle with multiple electrodes embedded along its shaft (Rutkove et al. 2018).

\section{CONCLUSIONS}

EIM remains a diagnostic technique of considerable promise with application across the neuromuscular disorders spectrum. As with any new technique, adoption has been slow, given that it takes a very different approach to muscle assessment than standard EMG or imaging modalities. In addition, most individuals in the medical community are fairly unfamiliar with the broader electrical impedance field itself, its potential value, and the challenging underlying concepts. Also, to a large extent, further work is required to standardize test procedures and data analysis approaches, a process that is likely to take many years. Nevertheless, EIM is gradually being adopted into clinical trials and further technological advancements, including easy-
Electrical Impedance in Neuromuscular Assessment

to-use systems and the advent of needle impedance-EMG, we believe, are likely to hasten acceptance in the coming years.

\section{ACKNOWLEDGMENTS}

This work was funded, in part, by National Institutes of Health (NIH) Grants K24 NS060951 and R01 NS091159.

\section{REFERENCES}

Aaron R, Esper GJ, Shiffman CA, Bradonjic K, Lee KS, Rutkove SB. 2006. Effects of age on muscle as measured by electrical impedance myography. Physiol Meas 27: 953-959.

Aberg P, Nicander I, Holmgren U, Geladi P, Ollmar S. 2003. Assessment of skin lesions and skin cancer using simple electrical impedance indices. Skin Res Technol 9: 257261.

Adler A, Amato MB, Arnold JH, Bayford R, Bodenstein M, Böhm SH, Brown BH, Frerichs I, Stenqvist O, Weiler N, et al. 2012. Whither lung EIT: Where are we, where do we want to go and what do we need to get there? Physiol Meas 33: 679-94.

Ahad M, Rutkove SB. 2009. Electrical impedance myography at $50 \mathrm{kHz}$ in the rat: Technique, reproducibility, and the effects of sciatic injury and recovery. Clin Neurophys 120: 1534-1538.

Ahad MA, Narayanaswami P, Kasselman LJ, Rutkove SB. 2010. The effect of subacute denervation on the electrical anisotropy of skeletal muscle: Implications for clinical diagnostic testing. Clin Neurophysiol 121: 882-886.

Arnold WD, McGovern VL, Sanchez B, Li J, Corlett KM, Kolb SJ, Rutkove SB, Burghes AH. 2016. The neuromuscular impact of symptomatic SMN restoration in a mouse model of spinal muscular atrophy. Neurobiol Dis 87: 116123.

Bozler E. 1935. The change of alternating current impedance of muscle produced by contraction. J Cell Comp Physiol 6: 217-228

Bozler E, Cole KS. 1935. Electric impedance and phase angle of muscle in rigor. J Cell Comp Physiol 6: 229-241.

Brown BH. 2003. Electrical impedance tomography (EIT): A review. J Med Eng Technol 27: 97-108.

Caresio C, Salvi M, Molinari F, Meiburger KM, Minetto MA. 2017. Fully automated muscle ultrasound analysis (MUSA): Robust and accurate muscle thickness measurement. Ultrasound Med Biol 43: 195-205.

Cole KS. 1928. Electric impedance of suspensions of spheres. J Gen Physiol 109: 29-36.

Ellis KJ. 2000. Human body composition: In vivo methods. Physiol Rev 80: 649-80.

Epstein BR, Foster KR. 1983. Anisotropy in the dielectric properties of skeletal muscle. Med Biol Eng Comput 21: 51-55. 
S.B. Rutkove and B. Sanchez

Foster KR, Schwan HP. 1989. Dielectric properties of tissues and biological materials: A critical review. Crit Rev Biomed Eng 17: 25-104.

Fricke H, Morse S. 1925. The electric resistance and capacity of blood for frequencies between 800 and $4 \frac{1}{2}$ million cycles. J Gen Physiol 9: 153-167.

Garmirian LP, Chin AB, Rutkove SB. 2009. Discriminating neurogenic from myopathic disease via measurement of muscle anisotropy. Muscle Nerve 39: 16-24.

Geddes LA, Baker LE. 1967. The specific resistance of biological material-A compendium of data for the biomedical engineer and physiologist. Med Biol Eng 5: 271-293.

Hakim CH, Mijailovic A, Lessa TB, Coates JR, Shin C, Rutkove SB, Duan D. 2017. Non-invasive evaluation of muscle disease in the canine model of Duchenne muscular dystrophy by electrical impedance myography. PLoS ONE 12: e0173557.

Holder DS. 1992. Electrical impedance tomography (EIT) of brain function. Brain Topogr 5: 87-93.

Holder DS. 2004. Electrical impedance tomography: Methods, history and applications. Institute of Physics, Bristol, UK.

Iannuzzi-Sucich M, Prestwood KM, Kenny AM. 2002. Prevalence of sarcopenia and predictors of skeletal muscle mass in healthy, older men and women. $J$ Gerontol $A$ Biol Sci Med Sci 57: M772-M777.

Janssen I, Heymsfield SB, Wang ZM, Ross R. 2000. Skeletal muscle mass and distribution in 468 men and women aged 18-88 yr. J Appl Physiol 89: 81-88.

Kapur K, Nagy JA, Taylor RA, Sanchez B, Rutkove SB. 2018a. Estimating myofiber size with electrical impedance myography: A study in ALS mice. Muscle Nerve doi: 10.1002/ mus.26187.

Kapur K, Taylor R, Qi K, Nagy J, Li J, Sanchez B, Rutkove S. 2018b. Predicting myofiber size with electrical impedance myography: A study in immature mice. Muscle Nerve doi: 10.1002/mus.26111.

Kemp GJ, Ahmad RE, Nicolay K, Prompers JJ. 2015. Quantification of skeletal muscle mitochondrial function by ${ }^{31} \mathrm{P}$ magnetic resonance spectroscopy techniques: A quantitative review. Acta Physiol 213: 107-144.

Kimura J. 2001. Electrodiagnosis in diseases of nerve and muscle: Principles and practice, 3rd ed. Oxford University Press, New York.

Kolb SJ, Coffey CS, Yankey JW, Krosschell K, Arnold WD, Rutkove SB, Swoboda KJ, Reyna SP, Sakonju A, Darras BT, et al. 2016. Baseline results of the NeuroNEXT spinal muscular atrophy infant biomarker study. Ann Clin Transl Neurol 3: 132-145.

Kortman HG, Wilder SC, Geisbush TR, Narayanaswami P, Rutkove SB. 2013. Age- and gender-associated differences in electrical impedance values of skeletal muscle. Physiol Meas 34: 1611-1622.

Kubicek WG, Patterson RP, Witsoe DA. 1970. Impedance cardiogaphy as a noninvasive method for monitoring cardiac function and other parameters of the cardiovascular system. Ann NY Acad Sci 170: 724-732.

Kwon H, Nagy JA, Taylor R, Rutkove SB, Sanchez B. 2017a. New electrical impedance methods for the in situ measurement of the complex permittivity of anisotropic biological tissues. Phys Med Biol 62: 8616-8633.
Kwon H, Rutkove SB, Sanchez B. 2017b. Recording characteristics of electrical impedance myography needle electrodes. Physiol Meas 38: 1748-1765.

Kwon H, Di Cristina JF, Rutkove SB, Sanchez B. 2018a. Recording characteristics of electrical impedance-electromyography needle electrodes. Physiol Meas 39: 55005.

Kwon H, Malik WQ, Rutkove SB, Sanchez B. 2018b. Separation of subcutaneous fat from muscle in surface electrical impedance myography measurements using model component analysis. IEEE Trans Biomed Eng doi: 10.1109/TBME.2018.2839977.

Kyle UG, Bosaeus I, De Lorenzo AD, Deurenberg P, Elia M, Gómez JM, Heitmann BL, Kent-Smith L, Melchior JC, Pirlich M, et al. 2004. Bioelectrical impedance analysis-Part I: Review of principles and methods. Clin Nutr 23: 1226-1243.

Li J, Staats WL, Spieker A, Sung M, Rutkove SB. 2012. A technique for performing electrical impedance myography in the mouse hind limb: Data in normal and ALS SOD1 G93A animals. PLoS ONE 7: e45004.

Li J, Geisbush TR, Rosen GD, Lachey J, Mulivor A, Rutkove SB. 2013a. Electrical impedance myography for the in and ex vivo assessment of muscular dystrophy $(m d x)$ mouse muscle. Muscle Nerve 49: 829-835.

Li J, Spieker AJ, Rosen GD, Rutkove SB. 2013b. Electrical impedance alterations in the rat hind limb with unloading. J Musculoskelet Neuronal Interact 13: 37-44.

Li J, Jafarpoor M, Bouxsein M, Rutkove SB. 2014. Distinguishing neuromuscular disorders based on the passive electrical material properties of muscle. Muscle Nerve 51: 49-55.

Li Z, Chen L, Zhu Y, Wei Q, Liu W, Tian D, Yu Y. 2017a. Handheld electrical impedance myography probe for assessing carpal tunnel syndrome. Ann Biomed Eng 45: 1572-1580.

Li Z, Dong T, Chen L, Wang X, Jiang L, Yu Y. 2017b. Electrical impedance myography for discriminating traumatic peripheral nerve injury in the upper extremity. Clin $\mathrm{Neu}$ rophysiol 128: 384-390.

Marchello MJ, Slanger WD. 1992. Use of bioelectrical impedance to predict leanness of Boston butts. J Anim Sci 70: $3443-3450$

McIlduff CE, Yim S, Pacheck A, Geisbush T, Mijailovic A, Rutkove SB. 2015. An improved electrical impedance myography tongue array for use in clinical trials. Clin Neurophysiol 127: 932-935.

Mul K, Heatwole C, Eichinger K, Dilek N, Martens WB, Van Engelen BGM, Tawil R, Statland JM. 2018. Electrical impedance myography in facioscapulohumeral muscular dystrophy: A 1-year follow-up study. Muscle Nerve 58: 213-218.

Nagy JA, Kapur K, Taylor RS, Sanchez B, Rutkove SB. 2018. Electrical impedance myography as a biomarker of myostatin inhibition with ActRIIB-mFc: A study in wild-type mice. Futur Sci OA 4: FSO308.

Nescolarde L. 2017. Detection of muscle gap by L-BIA in muscle injuries: Clinical prognosis. Physiol Meas 28: L1L9.

Nescolarde L, Yanguas J, Lukaski H, Alomar X, Rosell-Ferrer J, Rodas G. 2013. Localized bioimpedance to assess muscle injury. Physiol Meas 34: 237-245. 
Nescolarde L, Yanguas J, Lukaski H, Alomar X, Rosell-Ferrer J, Rodas G. 2015. Effects of muscle injury severity on localized bioimpedance measurements. Physiol Meas 36: $27-42$.

Phillips R. 2013. Prostate cancer: Increasing the potential of prostate biopsies with bioimpedance spectroscopy. Nat Rev Urol 10: 558.

Preston DC, Shaprio BE. 2005. Electromyography and neuromuscular disorders. Butterworth-Heinemann, Boston.

Rush S. 1962. Methods of measuring the resistivities of anisotropic conducting media in situ. J Res Natl Bur Stand Sect C Eng Instrum 66: 217.

Rutkove SB, Aaron R, Shiffman CA. 2002. Localized bioimpedance analysis in the evaluation of neuromuscular disease. Muscle Nerve 25: 390-397.

Rutkove SB, Esper GJ, Lee KS, Aaron R, Shiffman CA. 2005. Electrical impedance myography in the detection of radiculopathy. Muscle Nerve 32: 335-341.

Rutkove SB, Zhang H, Schoenfeld DA, Raynor EM, Shefner JM, Cudkowicz ME, Chin AB, Aaron R, Shiffman CA 2007. Electrical impedance myography to assess outcome in amyotrophic lateral sclerosis clinical trials. Clin Neurophysiol 118: 2413-2418.

Rutkove SB, Shefner JM, Gregas M, Butler H, Caracciolo J, Lin C, Fogerson PM, Mongiovi P, Darras BT. 2010. Characterizing spinal muscular atrophy with electrical impedance myography. Muscle Nerve 42: 915-921.

Rutkove SB, Caress JB, Cartwright MS, Burns TM, Warder J, David WS, Goyal N, Maragakis NJ, Clawson L, Benatar M, et al. 2012a. Electrical impedance myography as a biomarker to assess ALS progression. Amyotroph Lateral Scler 13: 439-445.

Rutkove SB, Gregas MC, Darras BT. 2012b. Electrical impedance myography in spinal muscular atrophy: A longitudinal study. Muscle Nerve 45: 642-647.

Rutkove SB, Geisbush TR, Mijailovic A, Shklyar I, Pasternak A, Visyak N, Wu JS, Zaidman C, Darras BT. 2014. Crosssectional evaluation of electrical impedance myography and quantitative ultrasound for the assessment of $\mathrm{Du}$ chenne muscular dystrophy in a clinical trial setting. Pediatr Neurol 51: 88-92.

Rutkove S, Murphy E, Halter R. 2017a. Imaging muscle contraction with electrical impedance. Annual Meeting of the American Association of Neuromuscular \& Electrodiagnostic Medicine. Phoenix, AZ, September 13-16.

Rutkove SB, Kapur K, Zaidman CM, Wu JS, Pasternak A, Madabusi L, Yim S, Pacheck A, Szelag H, Harrington T, et al. 2017b. Electrical impedance myography for assessment of Duchenne muscular dystrophy. Ann Neurol 81: 622-632.

Rutkove SB, Pacheck A, Sanchez B. 2017c. Sensitivity distribution simulations of surface electrode configurations for electrical impedance myography. Muscle Nerve 56: 887895.

Rutkove SB, Kwon H, Guasch M, Wu JS, Sanchez B. 2018. Electrical impedance imaging of human muscle at the microscopic scale using a multi-electrode needle device: A simulation study. Clin Neurophysiol 129: 1704-1708.

Sanchez B, Louarroudi E, Bragos R, Pintelon R. 2013a. Harmonic impedance spectra identification from time-vary- ing bioimpedance: Theory and validation. Physiol Meas 34: 1217-1238.

Sanchez B, Louarroudi E, Jorge E, Cinca J, Bragos R, Pintelon R. 2013b. A new measuring and identification approach for time-varying bioimpedance using multisine electrical impedance spectroscopy. Physiol Meas 34: 339-357.

Sanchez B, Li J, Bragos R, Rutkove SB. 2014a. Differentiation of the intracellular structure of slow- versus fast-twitch muscle fibers through evaluation of the dielectric properties of tissue. Phys Med Biol 59: 2369-2380.

Sanchez B, Li J, Geisbush T, Bragos R, Rutkove S. 2014b. Impedance alterations in healthy and diseased mice during electrically-induced muscle contraction. IEEE Trans Biomed Eng 63: 1602-1612.

Sanchez B, Li J, Yim S, Pacheck A, Widrick JJ, Rutkove SB. 2015a. Evaluation of electrical impedance as a biomarker of myostatin inhibition in wild type and muscular dystrophy mice. PLoS ONE 10: e0140521.

Sanchez B, Louarroudi E, Pintelon R. 2015b. Time-invariant measurement of time-varying bioimpedance using vector impedance analysis. Physiol Meas 36: 595-620.

Sanchez B, Pacheck A, Rutkove SB. 2016. Guidelines to electrode positioning for human and animal electrical impedance myography research. Sci Rep 6: 32615.

Sanchez B, Iyer SR, Li J, Kapur K, Xu S, Rutkove SB, Lovering RM. 2017. Non-invasive assessment of muscle injury in healthy and dystrophic animals with electrical impedance myography. Muscle Nerve 56: E85-E94.

Schwan HP. 1968. Electrode polarization impedance and measurements in biological materials. Ann NY Acad Sci 148: 191-209.

Schwan HP. 1999. The practical success of impedance techniques from an historical perspective. Ann NY Acad Sci 873: $1-12$.

Schwan HP, Kay CF. 1957. The conductivity of living tissues. Ann NY Acad Sci 65: 1007-1013.

Shefner JM, Rutkove SB, Caress JB, Benatar M, David WS, Cartwright MC, Macklin EA, Bohorquez JL. 2018. Assessing ALS progression with a dedicated electrical impedance myography system. Amyotroph Lateral Scler Frontotemporal Degener (in press).

Shellikeri S, Yunusova Y, Green JR, Pattee GL, Berry JD, Rutkove SB, Zinman L. 2015. Electrical impedance myography in the evaluation of the tongue musculature in amyotrophic lateral sclerosis. Muscle Nerve 52: 584-591.

Shiffman CA, Aaron R, Rutkove SB. 2003. Electrical impedance of muscle during isometric contraction. Physiol Meas 24: 213-234.

Silva OL, Sousa THS, Hoffman IO, de Camargo EDLB, de Moura FS, Martins ARC, Biasi C, Fantoni DT, Lima RG. 2014. A proposal to monitor muscle contraction through the change of electrical impedance inside a muscle. 5th IEEE RAS/EMBS International Conference on Biomedical Robotics and Biomechatronics. Sao Paulo, Brazil, August $12-15$.

Simon NG, Noto Y, Zaidman CM. 2016. Skeletal muscle imaging in neuromuscular disease. J Clin Neurosci 33: 1-10.

Spieker AJ, Narayanaswami P, Fleming L, Keel JC, Muzin SC, Rutkove SB. 2013. Electrical impedance myography in the diagnosis of radiculopathy. Muscle Nerve 48: 800-805. 
S.B. Rutkove and B. Sanchez

Statland JM, Heatwole C, Eichinger K, Dilek N, Martens WB, Tawil R. 2016. Electrical impedance myography in facioscapulohumeral muscular dystrophy. Muscle Nerve 54: 696-701.

Stoy RD, Foster KR, Schwan HP. 1982. Dielectric properties of mammalian tissues from 0.1 to $100 \mathrm{MHz}$ : A summary of recent data. Phys Med Biol 27: 501-513.

Sung M, Li J, Spieker AJ, Spatz J, Ellman R, Ferguson VL, Bateman TA, Rosen GD, Bouxsein M, Rutkove SB. 2013. Spaceflight and hind limb unloading induce similar changes in electrical impedance characteristics of mouse gastrocnemius muscle. J Musculoskelet Neuronal Interact 13: 405-411

Tarulli A, Esper GJ, Lee KS, Aaron R, Shiffman CA, Rutkove SB. 2005. Electrical impedance myography in the bedside assessment of inflammatory myopathy. Neurology 65: 451-452.

Tarulli AW, Duggal N, Esper GJ, Garmirian LP, Fogerson PM, Lin CH, Rutkove SB. 2009a. Electrical impedance myography in the assessment of disuse atrophy. Arch Phys Med Rehabil 90: 1806-1810.

Tarulli AW, Garmirian LP, Fogerson PM, Rutkove SB. 2009b. Localized muscle impedance abnormalities in amyotrophic lateral sclerosis. J Clin Neuromuscul Dis 10: $90-96$.

Toso S, Piccoli A, Gusella M, Menon D, Bononi A. 2000 Altered tissue electric properties in lung cancer patients as detected by bioelectric impedance vector analysis. Nutrition 16: 120-124.

Urso ML, Clarkson PM, Price TB. 2006. Immobilization effects in young and older adults. Eur J Appl Physiol 96: 564-571.

Wang LL, Spieker AJ, Li J, Rutkove SB. 2011. Electrical impedance myography for monitoring motor neuron loss in the SOD1 G93A amyotrophic lateral sclerosis rat. Clin Neurophysiol 122: 2505-2511.

Willcocks RJ, Rooney WD, Triplett WT, Forbes SC, Lott DJ, Senesac CR, Daniels MJ, Wang DJ, Harrington AT, Tennekoon GI, et al. 2016. Multicenter prospective longitudinal study of magnetic resonance biomarkers in a large Duchenne muscular dystrophy cohort. Ann Neurol 79: 535-547.

Wu JS, Li J, Greenman RL, Bennett D, Geisbush T, Rutkove SB. 2015. Assessment of aged $m d x$ mice by electrical impedance myography and magnetic resonance imaging. Muscle Nerve 52: 598-604.

Zagar T, Krizaj D. 2008. Multivariate analysis of electrica impedance spectra for relaxed and contracted skeletal muscle. Physiol Meas 29: S365-S372.

Zaidman CM, Wang LL, Connolly AM, Florence J, Wong BL, Parsons JA, Apkon S, Goyal N, Williams E, Escolar D, et al. 2015. Electrical impedance myography in Duchenne muscular dystrophy and healthy controls: A multicenter study of reliability and validity. Muscle Nerve 52: 592-597.

Zaidman CM, Wu JS, Kapur K, Pasternak A, Madabusi L, Yim S, Pacheck A, Szelag H, Harrington T, Darras BT, et al. 2017. Quantitative muscle ultrasound detects disease progression in Duchenne muscular dystrophy. Ann Neurol 81: 633-640.

Zaidman CM, Kapur K, Darras BT, Wong BL, Yang ML, Leitner ML, Dalle Pazze L, Buck M, Freedman L, Rutkove SB. 2018. Insights from a multisite study utilizing dedicated technology to assess electrical impedance myography as an outcome measure for Duchenne muscular dystrophy. World Muscle Society Congress 2018. Mendoza, Argentina, October 2-6. 


\title{
$\&_{\mathrm{CSH}}^{\infty} \&$ Cold Spring Harbor

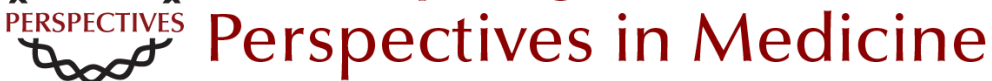

\section{Electrical Impedance Methods in Neuromuscular Assessment: An Overview}

\author{
Seward B. Rutkove and Benjamin Sanchez
}

Cold Spring Harb Perspect Med 2019; doi: 10.1101/cshperspect.a034405 originally published online October 5, 2018

\section{Subject Collection Bioelectronic Medicine}

Neural Control of Inflammation: Bioelectronic Medicine in Treatment of Chronic Inflammatory Disease

Michael Eberhardson, Laura Tarnawski, Monica Centa, et al.

Noninvasive Neuromodulation of Peripheral Nerve Pathways Using Ultrasound and Its Current

Therapeutic Implications

Christopher Puleo and Victoria Cotero

Enteric Neuromodulation for the Gut and Beyond Yogi A. Patel and Pankaj J. Pasricha

Optogenetic Control of the Peripheral Nervous System

Rui B. Chang

Closed-Loop Neuromodulation in Physiological and Translational Research

Stavros Zanos

Electrical Impedance Methods in Neuromuscular Assessment: An Overview

Seward B. Rutkove and Benjamin Sanchez

Optogenetic Medicine: Synthetic Therapeutic

Solutions Precision-Guided by Light

Haifeng Ye and Martin Fussenegger

Technobiology's Enabler: The Magnetoelectric Nanoparticle

Sakhrat Khizroev
Bioelectronic Medicine: From Preclinical Studies on the Inflammatory Reflex to New Approaches in Disease Diagnosis and Treatment

Valentin A. Pavlov, Sangeeta S. Chavan and Kevin

J. Tracey

Vagus Nerve Stimulation and the Cardiovascular System

Michael J. Capilupi, Samantha M. Kerath and

Lance B. Becker

Harnessing the Inflammatory Reflex for the

Treatment of Inflammation-Mediated Diseases

Yaakov A. Levine, Michael Faltys and David Chernoff

Recording and Decoding of Vagal Neural Signals Related to Changes in Physiological Parameters and Biomarkers of Disease Theodoros P. Zanos

Restoring Movement in Paralysis with a

Bioelectronic Neural Bypass Approach: Current

State and Future Directions Chad E. Bouton

Bioelectronic Medicine--Ethical Concerns Samuel Packer, Nicholas Mercado and Anita Haridat

Use of Bioelectronics in the Gastrointestinal Tract Larry Miller, Aydin Farajidavar and Anil Vegesna

Vagus Nerve Stimulation at the Interface of BrainGut Interactions

Bruno Bonaz, Valérie Sinniger and Sonia Pellissier

For additional articles in this collection, see http://perspectivesinmedicine.cshlp.org/cgi/collection/ 